\title{
Editorials
}

\section{Analysis of forced expiration-a return to the recording spirometer?}

The dominant functional abnormality in intrapulmonary airflow obstruction is a slowing of the maximum rate of expiration. For the past 30 years, since the introduction of the analysis of the forced expiratory spirogram by Tiffeneau and Pinelli (1947), there has been a continuous effort to define the best way to extract information from this simple manoeuvre. The first emphasis was on measurements of expired volume at standard times, such as the forced expiratory volume in one second $\left(\mathrm{FEV}_{1}\right)$. The $\mathrm{FEV}_{1}$ was chosen empirically, because it correlated well with an earlier test, the maximum breathing capacity (maximum voluntary ventilation sustained over 15 seconds), and was simpler for subject and investigator. That an expiratory test correlated so well with the maximum breathing capacity emphasised the dominant role of expiratory flow limitation in determining maximum ventilation, and in the nineteen-fifties it became possible to measure flow directly either with a pneumotachograph or with an even simpler device, the peak flow meter (Wright and McKerrow, 1959). The major criticism of all flow measurements was that they depended on effort but the driving pressure was unknown; this criticism still inhibits the use of peak flow fully outside Britain. Physiologists preferred to measure the relation between driving pressure (difference between alveolar and mouth pressure) and flow (that is airways resistance), which had also become a practical technique after the introduction of the body plethysmograph in 1956 . For some years it appeared that airflow obstruction should be defined as an increased resistance to airflow (Ciba Guest Symposium, 1959) but, apart from the practical problem that the body plethysmograph was suitable only for use in specialised laboratories and was expensive, it soon became clear that resistance varied considerably with laryngeal aperture, airflow rate, and lung volume. Variation was particularly pronounced on expiration in patients with airway disease, so that the measurement that was most wanted was the most difficult to obtain.

It was fortunate that the comprehensive analysis of the relation between pressure, flow, and lung volume made by Fry and Hyatt (1960) showed that precise knowledge of the driving pressure during a forced expiration was not so important as had been expected. Fry and Hyatt showed that, at a given lung volume, expiratory flow increased with increased pressure only at low driving pressure; as the pressure was increased further, expiratory flow remained at a nearly constant level regardless of the pressure. In addition to showing that flow at a given volume can be nearly entirely independent of pressure and expiratory effort (especially at small lung volume), they showed that maximum expiratory flow was noticeably dependent on the lung volume, so that the larger the lung volume, the larger the flow. Thus airflow obstruction could be regarded as a decrease in maximum flow at a given volume, and it appeared that the maximum expiratory flow-volume curve could be the best method for expressing the events of forced expiration. Interest in the flow-volume curve was strengthened by the important work of Mead, Macklem, and co-workers (1967), which indicated that in normal lungs maximum expiratory flow at large lung volumes, such as peak flow, depended mainly on the dimensions of the central intrathoracic airways, while maximum flow at small lung volumes, especially below the resting breathing position, reflected predominantly the function of the peripheral airways. As the simple spirometric tests such as the $\mathrm{FEV}_{1}$ and $\mathrm{FEV}_{1} / \mathrm{VC}$ ratio (and even more peak flow) reflected flow at volumes above the resting breathing position they could be regarded (in normal subjects) as tests of large airway function. When Macklem and his colleagues in Montreal (Hogg et al, 1968; Macklem, 1972) went on to show that the early changes in chronic airway disease were in the peripheral airways it became essential to measure events in the terminal quarter of expiration, and this enhanced the potential value of measurements of maximum flow at small lung volumes, at least for those interested in the detection of minor abnormalities of airway function.

The major problem in using the flow-volume curve has been that variation in normal airway function and anatomy is large so that the reference standards for maximum flow at a given lung volume have been wide, and it is correspondingly difficult to detect early changes due to disease. 
The introduction of the comparison of flow-volume curves breathing air and a helium-oxygen mixture (Dosman et al, 1975) therefore was a useful advance; by studying the change in maximum flow at a given volume, differences in original airway anatomy and function are largely allowed for and a reduction in the flow response to helium breathing shows promise as an indicator of early disease of the peripheral airways. One disappointment with the flow-volume curve is that it has proved difficult to obtain a simple, quantitative measurement of the curvilinearity (decreasing flow/volume slope with decreasing lung volume) that is such a characteristic feature of the curve in intrapulmonary airflow obstruction.

We can now see that there has been a logical progression from reliance on volume alone, to timed volume measurements, to direct measurement of flow (volume/time), and on to pressure/ flow (resistance) and flow as a function of volume. The discovery of the relative independence of maximum flow from the effort applied (even at large lung volumes, peak flow, although "effortdependent," is repeatable, indicating that effort does not vary that much), means that the pressure applied during forced expiration can be effectively ignored so that the original concept of Tiffeneau of describing only volume versus time is justified. But what we have learnt since 1947 is that there is much information-if only we can quantify itin the terminal part of the spirogram, so that the present received view is that FEV $_{1}$, peak flow, and $\mathrm{FEV}_{1} / \mathrm{VC}$ are insensitive tests for detecting minor abnormalities of airway function when the affected airways lie in the periphery of the lung.

At least ten different measurements of the forced expiratory manoeuvre are in widespread use today, and others have had earlier periods of popularity. So a considerable responsibility falls on investigators who propose further ways of analysing this manoeuvre. Yet it is clear that this intensive effort has concentrated almost entirely on volume and flow measurements, while time has been largely ignored, even although the primary functional abnormality is well recognised to be a slowing of forced expiration. Measurement of the time to complete a forced expiration (forced expiratory time) was developed by Rosenblatt and Stein (1962) and has proved useful in distinguishing between normal subjects and those with moderate or severe airflow obstruction, but it has been difficult to refine the test. More recently Permutt, Menkes, and colleagues in Baltimore (Tockman et al, 1976; Permutt and Menkes, 1979) have described the use of the mean transit time of forced expiration. They have adapted the well-established principles of obtaining mean transit time of an injected indicator in the circulation to obtain the mean transit time for the transfer of gas molecules from alveoli to the atmosphere during a forced expiration. In subjects with normal lungs most gas molecules are transferred rapidly and with short transit times from alveoli to mouth, while some gas molecules require a much longer time for transfer and these have long transit times. The mean transit time of a forced expiration can be readily obtained by integrating the area under the spirogram curve of volume versus time and dividing by the forced vital capacity (FVC) (see figure). This can be done by a simple computer program. In theory mean transit time depends on events throughout the forced expiration and is not limited to measurements made during specific parts of the expiration. In practice, however, mean transit time is particularly sensitive to the presence of an increased population of slowly emptying units with long transit times, while an increased proportion of rapidly emptying units will not produce striking reductions in mean transit time. In the light of present knowledge of the evolution of intrapulmonary airflow obstruction these characteristics of the volume-time curve should make it a highly suitable method for detecting early

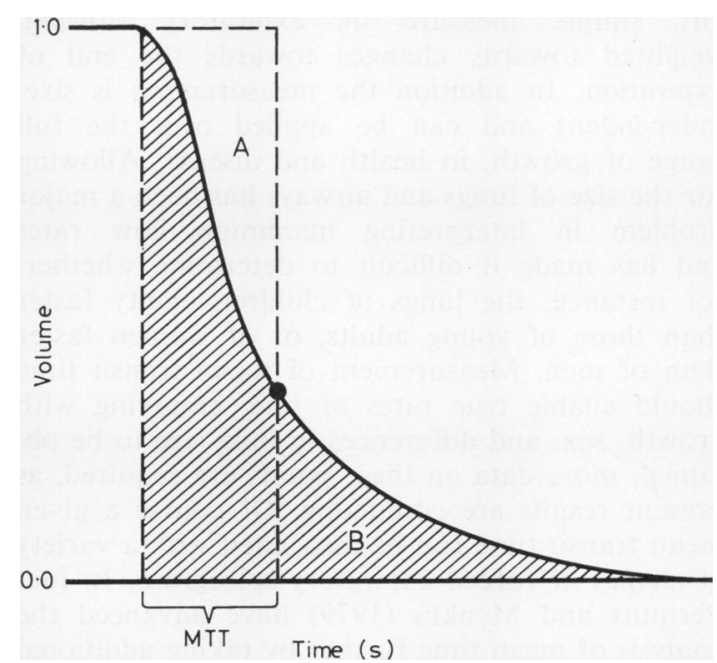

Analysis of forced expired spirogram (change in volume versus time) to obtain mean transit time (MTT) of expiration. When volume change (forced vital capacity) is normalised, as shown, area under spirogram (diagonal shaded area) is MTT. Shaded area is equivalent to rectangular area indicated by dashed lines (that is, unshaded area $(A)$ within dashed rectangle $=$ shaded area $(B)$ lying to right of dashed rectangle). Where dashed rectangle intersects time axis indicates value of $M T T$. 
changes. In contrast the rival flow-volume technique is extremely valuable for examining early events in expiration, because pronounced changes in flow occur within the first fraction of a second, where these events are very compressed on the time axis of the spirogram. But with the flowvolume analysis all the late events in time are very compressed on the extreme end of the curve (Permutt and Menkes, 1979).

Two papers in this issue of Thorax confirm that measuring mean transit time is a promising method for detecting early changes in the peripheral airways. Each group has analysed the spirogram by the technique introduced by the Johns Hopkins workers, but in the paper by Jordanoglou and colleagues mean transit time has been termed "effective time" ( $t$ eff). Jordanoglou shows that smokers with chronic expectoration and $\mathrm{FEV}_{1} /$ FVC ratios in the normal range often have increased mean transit times. Liang and colleagues from Auckland find that mean transit time is more frequently abnormal in asthmatic children in remission than $\mathrm{FEV}_{1} / \mathrm{FVC}$ and, more importantly, than maximum flow in the lower half of the FVC.

What are the advantages and disadvantages of analysing the mean transit time of the spirogram? Most obviously it provides a direct and conceptually simple measure of expiratory slowing, weighted towards changes towards the end of expiration. In addition the measurement is sizeindependent and can be applied over the full range of growth, in health and disease. Allowing for the size of lungs and airways has been a major problem in interpreting maximum flow rates and has made it difficult to determine whether, for instance, the lungs of children empty faster than those of young adults, or of women faster than of men. Measurement of mean transit time should enable true rates of lung emptying with growth, sex, and differences in lung size to be obtained; more data on these points are required, as present results are conflicting. Of course a given mean transit time can be associated with a variety of shapes of forced expiratory spirogram. In fact Permutt and Menkes (1979) have advanced the analysis of mean time further by taking additional moments of the spirogram that enable the standard deviation of transit times and the skewness of the distribution of transit times to be calculated also. This has both mathematical and physiological advantages. Mathematically, it enables them to describe the precise shape of a forced expiratory spirogram by a few parameters. Physiologically, the higher moments are even more sensitive to the terminal part of the spirogram than mean transit time and provide evidence on the uniformity of lung emptying. Two major disadvantages are so far apparent. The first is that the results are sensitive to how long the expiration is continued (this was also a major problem with forced expiratory time) but Permutt has neatly sidestepped this by analysing data to six seconds and then extrapolating the spirogram mathematically to infinity. It is particularly important to avoid or correct for truncation of the curve if higher moments of the spirogram are analysed. The second and very obvious disadvantage is that, although the procedure is conceptually simple, integration of the area under the curve (and even more analysis of higher moments) requires computation, but this problem should rapidly decline with the development of microprocessor techniques.

This further refinement of the analysis of the forced expiratory spirogram is intriguing on several counts. For those interested in basic principles it is reassuring that elaborate analysis of pressure, flow, and volume interrelations has facilitated a return to simplicity; not just a full circle but returning to the spirogram with improved understanding of how to extract from it information about the function of both large and small airways and the uniformity of lung emptying. For the epidemiologist it raises the possibility that a more sophisticated analysis of the simple forced spirogram will help in discriminating between slightly low values of $F E V_{1}$ that are due to disease and those due to anatomical differences in the size of the lungs without having to employ additional tests of small airway function. For those who relied empirically on the spirogram, it provides reassurance that, if accurately recorded and analysed, it can show all the information contained in the later parts of the flow-volume curve. And finally, for those who think "it has all been done" it must be somewhat surprising to find that, after 30 years of sophisticated research and intensive practical application, something as obvious as analysis of the time of forced expiration had escaped rigorous investigation.

N B PRIDE

Royal Postgraduate Medical School Hammersmith Hospital, London W12

\section{References}

Ciba Guest Symposium (1959). Terminology, definitions, and classification of chronic pulmonary emphysema and related conditions. Thorax, 14, 286-299.

Dosman, J, Bode, F, Urbanetti, J, Martin, R, and 
Macklem, P T (1975). The use of a helium-oxygen mixture during maximum expiratory flow to demonstrate obstruction in small airways in smokers. Journal of Clinical Investigation, 55, 1090-1099.

Fry, D L, and Hyatt, R E (1960). Pulmonary mechanics. American Journal of Medicine, 29, 672-689.

Hogg, J C, Macklem, P T, and Thurlbeck, W M (1968). Site and nature of airway obstruction in chronic obstructive lung disease. New England Journal of Medicine, 278, 1355-1360.

Macklem, P T (1972). Obstruction in small airwaysa challenge to medicine. American Journal of Medicine, 52, 721-724.

Mead, J, Turner, J M, Macklem, P T, and Little, J B (1967). Significance of the relationship between lung recoil and maximum expiratory flow. Journal of Applied Physiology, 22, 95-108.

Permutt, S, and Menkes, H A (1979). Spirometry: analysis of forced expiration within the time domain. In The Lung in the Transition between
Health and Disease, chapter 6 (Lung biology in health and disease series, vol 12), edited by $P$ Macklem and S Permutt. Marcel Dekker, New York.

Rosenblatt, G, and Stein, M (1962). Clinical value of the forced expiratory time measured during auscultation. New England Journal of Medicine, 267, 432435.

Tiffeneau, R, and Pinelli, A (1947). Air circulant et air captif dans l'exploration de la fonction ventilatrice pulmonaire. Paris Médical, 37, 624-628.

Tockman, M, Menkes, H, Cohen, B, Permutt, S, Benjamin, J, Ball, W C, jun, and Tonascia, J (1976). A comparison of pulmonary function in male smokers and non-smokers. American Review of Respiratory Disease, 114, 711-722.

Wright, B M, and McKerrow, C B (1959). Maximum forced expiratory flow rate as a measure of ventilatory capacity with a description of a new portable instrument for measuring it. British Medical Journal, 2, 1041-1047.

\section{Digoxin for heart failure in sinus rhythm}

Is digoxin useful in patients with heart failure who remain in sinus rhythm? This question is still being asked and may reflect the profound influence that MacKenzie and Lewis have had on British medicine. Generations of medical students were taught that digitalis was of little value unless the patient was in atrial fibrillation; it is certainly true that in their earlier writings both MacKenzie (1913) and Lewis (1933) avoided discussing the problem of the patient with heart failure in sinus rhythm. However, the beneficial effect of digitalis in these patients was shown in several studies (Christian, 1919; Harrison et al, 1931; Gavey and Parkinson, 1939; Wood, 1940), and by 1946 Lewis was able to write "Clear evidence that digitalis is useful in failure with congestion presenting normal rhythm has been slower in coming because success in such cases is less obvious and less frequent. Nevertheless it is now generally recognised that digitalis is valuable in these cases and sometimes succeeds in lowering the venous pressure and in bringing relief where other remedies have failed." But despite this, clinicians remained reluctant to use digoxin in these circumstances, no doubt because they had been taught otherwise at an impressionable age.

The fall in venous pressure and rise in cardiac output resulting from intravenous digoxin in patients with heart failure was confirmed by McMichael and Sharpey-Shafer (1944). The cardiac output fell in normal subjects and in those with cor pulmonale, and at that time it was thought the primary action of digoxin was to lower the venous pressure. Although these authors subsequently revised their opinion, the difficulty in showing a positive inotropic effect with the techniques then available led McMichael to state in 1952 "Digitalis has no measurable effect on the contractile force of a normally functioning myocardium" (McMichael, 1952). This statement is still remembered and, moreover, the idea that digoxin might actually be harmful to patients with cor pulmonale (Howarth et al, 1948) means that its use is often restricted to those patients with atrial fibrillation.

The development of more sensitive techniques for measuring the force of ventricular contraction in man allowed Braunwald and his colleagues to show the positive inotropic effect of digoxin on the non-failing human heart by various means, including strain gauges attached to the myocardium during cardiac operations (Braunwald et al, 1961), the measurement of peak left ventricular $\mathrm{dp} / \mathrm{dt}$ using a catheter tip manometer (Mason and Braunwald, 1963) and the use of ventricular markers to study myocardial force-velocity relations (Sonnenblick et al, 1966). Nowadays the acute positive inotropic effect of digoxin on both the failing and non-failing human heart cannot be doubted. The measurement of systolic time intervals and the ball travel time of an aortic Starr valve also reflect the contractile state of the left 